\title{
Development of Combined Silicon Plate Nozzles*
}

\author{
Yasuhide TANI $^{* *}$, Hideto INAGAKI ${ }^{* * *}$, \\ Akinori SAITO $^{* * *}$ and Takashi SUZUKI ${ }^{* * * *}$
}

\begin{abstract}
Exhaust emission and fuel consumption of a vehicle engine can be reduced by improving fuel atomization of an injection nozzle. Authors have already reported development of a new type of nozzle called Silicon Plate Nozzle (SPN) in order to improve fuel atomization by adopting micro-machining. This paper discusses combination of two SPNs which resulted in drastic improvement of fuel atomization. By combining two SPNs so that each rectangular aperture crossed with other, the fan-like spray was obtained. As a result, obtained Sauter Mean Diameter (SMD) of the fuel spray was $50 \mu \mathrm{m}$ or less at fuel pressure of $300 \mathrm{kPa}$. This experimental results were confirmed by numerical analysis.
\end{abstract}

Key Words: Nozzle, Fuel Injection, Atomization, Liquid Fuel, Gasoline Engine, Silicon Plate Nozzle

\section{Introduction}

To meet the demand for cleaner exhaust emissions and lower fuel consumption in vehicle engines, it has become increasingly important to improve the fuel supply system. Reduction of hydrocarbon (HC) emission and suppression of fuel injection increase at cold start are particularly important challenges. These challenges can be effectively addressed by improving the fuel atomization performance of the injection nozzle. For example, our past study has confirmed that an air-assist injector, which adds air flow in the fuel to improve atomization, can substantially reduce $\mathrm{HC}$ emission ${ }^{(1)}$. A multiple-hole plate nozzle, which enhances turbulence near the nozzle orifice to improve fuel atomization, has also been confirmed to have the same or higher $\mathrm{HC}$ emission reduction effect as/than the air-assist injector ${ }^{(2)}$. As described in our study report $^{(2)}$, however, the air-assist injector provides superior atomization performance only under a light load, due to the pressure condition in the air intake pipe. The multiplehole plate nozzle as well leaves room for further improve-

* Received 23rd July, 2004 (No. 02-0998). Japanese Original: Trans. Jpn. Soc. Mech. Eng., Vol.69, No.681, B(2003), pp.1290-1295 (Received 9th August, 2002)

** DENSO CORPORATION, 1-1 Showa-cho, Kariya-shi, Aichi 448-8661, Japan.

E-mail: yasuhide_tani@denso.co.jp

*** Toyota Central R\&D Labs., Inc., 41-1 Yokomichi, Nagakute, Nagakute-cho, Aichi-gun, Aichi 480-1192, Japan

**** Toyohashi University of Technology, 1-1 Hibarigaoka, Tenpaku-cho, Toyohashi-shi, Aichi 441-8580, Japan ment of fuel atomization.

Against this background, we have been researching development of a new type of injection nozzle using micro-machining technology with the aim of enhancing fuel atomization. The new nozzle comprises an orifice formed in a silicon wafer, and is called a silicon plate nozzle (hereinafter referred to as "SPN"). The basic construction of the SPN has already been reported ${ }^{(3)}$. In that report, we discussed the influence which the turbulent intensity of flow near the nozzle orifice can exert on the atomization characteristic. In an attempt to increase the turbulent intensity near the orifice, we experimentally combined two SPNs each having a rectangular orifice formed in it. When two SPNs were superposed so that the orifices crossed at right angles with each other, the spray pattern changed suddenly to become a flat, fan-shaped spray. Further experiments revealed that the spray characteristics change widely with the orifice configuration and the crossing angles of the two orifices.

We studied the spray characteristics of the newly developed injection nozzle in which two SPNs are combined (hereinafter referred to as the "combined SPN"), as well as the fan-spray formation mechanism of the combined SPN through three-dimensional numerical analysis of the nozzle fuel flow. This report presents the results of these studies.

\section{Construction of Combined SPN}

The basic construction of a single SPN element used in the combined SPN is the same as described in our previous report ${ }^{(3)}$. It comprises a silicon wafer in which a 
nozzle orifice is formed by etching. Specifically, as shown in Fig. 1, after a nitride layer is created on both sides of a silicon wafer, the portion of layer on one side where a nozzle orifice is to be formed is removed by photo lithography and reactive ion etching. Next, the wafer is etched anisotropically using potassium hydroxide until the nitride layer on the other side is exposed. Finally, the nitride layers on both sides are removed to complete a nozzle orifice in the silicon wafer. Due to the characteristics of anisotropic etching, the angle of the sloped wall surrounding the nozzle orifice is $54.7^{\circ}$ as shown in Fig. 1 . The orifice plate thus produced is $0.36 \mathrm{~mm}$ in thickness.

Figure 3 shows the basic construction of the combined SPN. Two SPN elements each having a rectangular orifice shown in Fig. 2 are superposed so that the orifices cross at right angles with each other. Experiments were carried out to evaluate the spray characteristics of the combined SPN for various combinations of nozzle orifice specifications: all six specifications shown in Fig. 2 for the upstream orifice, and three specifications selected from Fig. 2 for the downstream orifice. Table 1 gives the

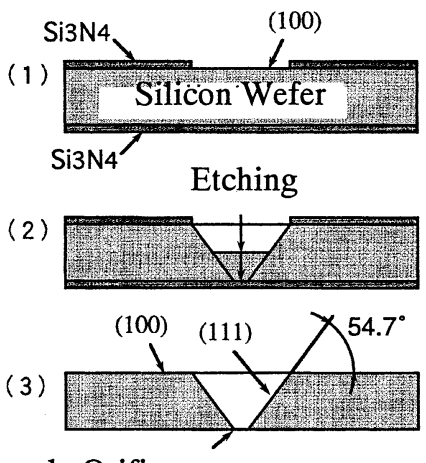

Nozzle Orifice

Fig. 1 Silicon nozzle etching process

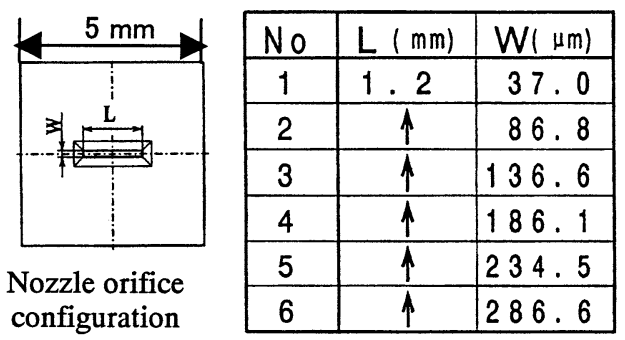

Fig. 2 Nozzle orifice specifications for combined SPN

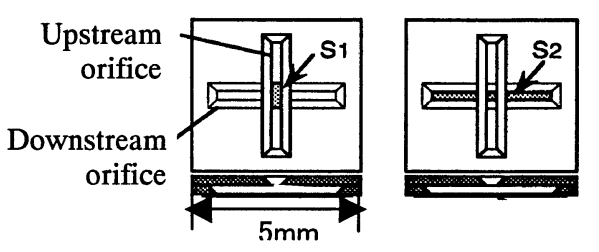

S1: upstream orifice area, S2: downstream orifice area

Fig. 3 Basic constraction of combined SPN orifice area ratio of each combination.

\section{Method for Measuring Spray Characteristics}

With the combined SPN mounted in a nozzle holder as shown in Fig. 4, fuel was supplied under a constant pressure to the combined SPN, to study the spray characteristics.

In the experiment, unleaded gasoline was used. The fuel pressure was $300 \mathrm{kPa}$, which was the same pressure as for the conventional electronic fuel injection (EFI) injector. Fuel temperature and nozzle ambient temperature were set at the room temperature. The spray characteristics were evaluated regarding the following two items:

(1) The spray pattern observed by instant photography using a xenon flash lamp (Flash duration $<1 \mu$ s)

(2) The spray droplet distribution and mean droplet size measured by the laser diffraction method

Figure 5 shows the details for measuring the spray droplet distribution by the laser diffraction method. The measurement apparatus used was a laser droplet size analyzer LDSA-1300A manufactured by Tonichi Computer Applications Co., Ltd. This analyzer emits a laser beam of $10 \mathrm{~mm}$ in diameter and provides a measuring range of 0.9 to $255 \mu \mathrm{m}$. To enable measurement of droplet distribution over the entire fan spray, spray was slanted with respect to the laser beam as shown in Fig. 5, and the analyzer was moved at 5-mm intervals. The droplet distributions obtained at all measuring positions were summed up to calculate the mean droplet size. Fuel injection pressure was constant as mentioned above. The fan spray angle varied depending on the combination nozzle orifice specifications. The experimental result verified that it is possible

Table 1 Combinatin of two SPNs and orifice area ratio

\begin{tabular}{c|c|c|c}
\hline Upstream orifice & \multicolumn{3}{|c}{ Downstream orifice width $(\mu \mathrm{m})$} \\
\cline { 2 - 4 } width $(\mu \mathrm{m})$ & 86.8 & 136.6 & 186.1 \\
\hline 37.0 & 0.21 & 0.15 & 0.12 \\
86.8 & 0.50 & 0.34 & 0.27 \\
136.6 & 0.78 & 0.54 & 0.42 \\
186.1 & 1.06 & 0.73 & 0.58 \\
234.5 & 1.34 & 0.92 & 0.73 \\
286.6 & 1.64 & 1.13 & 0.89 \\
\hline
\end{tabular}

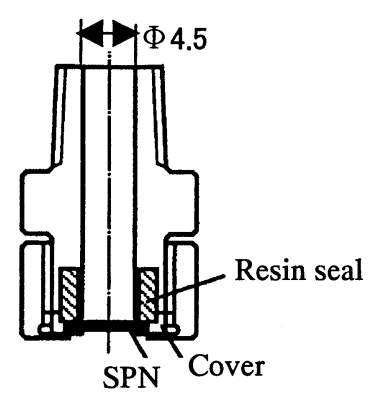

Fig. 4 Experimental assembly for SPN (constant pressure injection) 


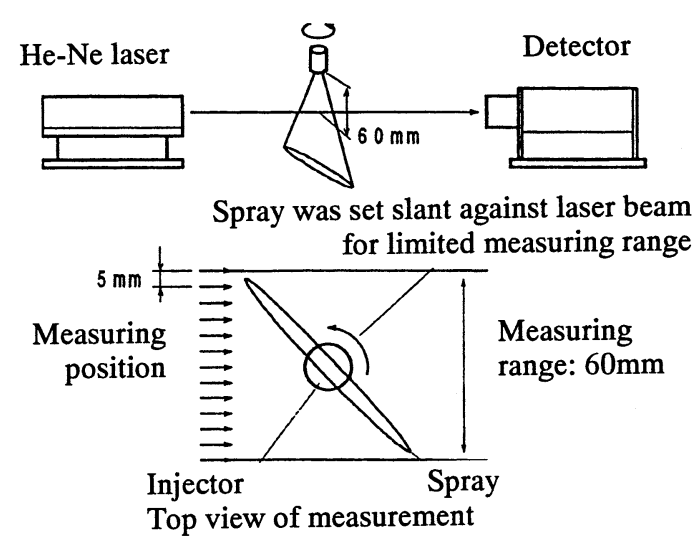

Fig. 5 Spray droplet distribution measurement with laser diffraction method

to measure the fuel droplet distribution and mean droplet size in any spray produced by the combined SPN in the measuring range specified in Fig. 5.

\section{Spray Characteristics of Combined SPN}

\subsection{Spray pattern}

Figure 6 shows the typical spray pattern produced by the combined SPN that uses orifice specification No. 2 given in Fig. 2 for both the upstream and downstream orifices. A fan-shaped spray pattern was produced. The upper picture of Fig. 6 shows the front image of the spray. For this image, the spray was illuminated obliquely with a flash light positioned at a point slightly to the right of the spray, and the scattered light reflected from the spray was photographed. The lower picture of Fig. 6 shows the cross sectional image of the spray. For this image, the spray was illuminated with a slit light positioned at a point $60 \mathrm{~mm}$ below the nozzle orifice, and the scattered light reflected from the spray was photographed obliquely from above.

As shown in Fig. 7, the spray characteristics of the combined SPN vary according to the downstream orifice width and the orifice area ratio (the ratio of upstream orifice area to downstream orifice area) specified in Fig. 3. When the orifice area ratio is extremely low, the combined SPN provides insufficient fan spray formation and atomization. Also too high orifice area ratio results in slight decrease in the fan spray angle. The spray characteristics change suddenly in the vicinity of the orifice area ratios with 0.3 and 0.7 .

When the orifice area ratio is lower than 0.3 , the combined SPN produces spray consisting of three jet flows with the liquid film concentrated in the center flow. With this orifice area ratio, since fuel flows at a low rate from the upstream to downstream orifice, the velocity of fuel injection from the downstream orifice is low. The consequence is that the film of fuel liquid ruptures before it extends sufficiently downward. Presumably, this is why the fuel is sprayed in three jet flows: the center flow straight under the upstream orifice, and two branch flows on both

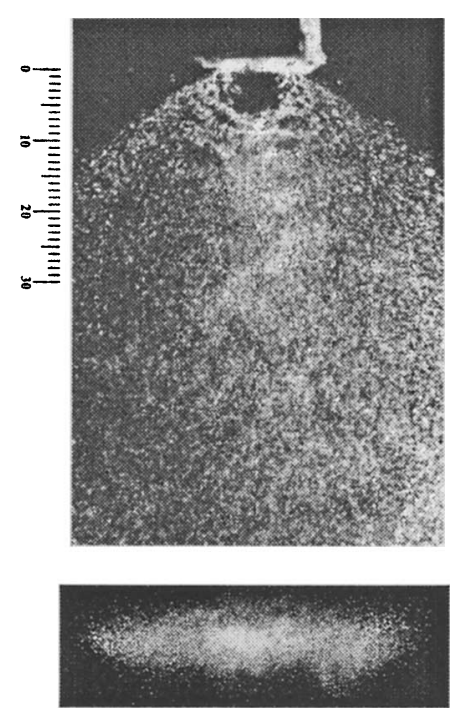

Fig. 6 Typical spray pattern of combined SPN (injection pressure: $300 \mathrm{kPa}$ )

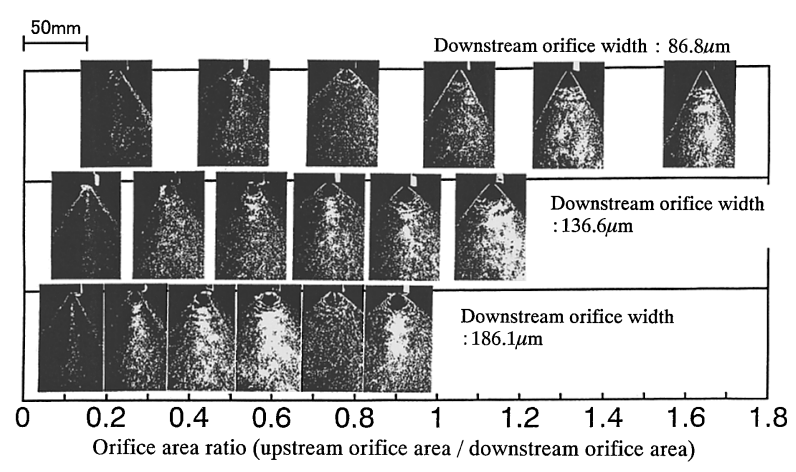

Fig. 7 Spray pattern changes on orifice area ratio (injection pressure: $300 \mathrm{kPa}$ )

sides of the center flow.

When the orifice area ratio is between 0.3 and 0.7 , the combined SPN produces a favorable spray pattern almost uniform in atomization over the entire spray. When the orifice area ratio exceeds 0.7 , high-velocity branch flows are formed on both sides of the fan spray. With this orifice area ratio, since fuel flows at a high rate from the upstream to downstream orifice, the fuel fills up the downstream orifice, possibly producing a high-velocity branch flow on each side of the fan spray. The side branch flows thus produced constrain fan formation, resulting in decreased fan spray angle.

Figure 8 shows the fuel flow rate characteristics of the combined SPN. The fuel flow rate decreases with the downstream orifice width. Regardless of the downstream orifice width, the fuel flow rate increases almost linearly with the orifice area ratio until the ratio reaches 0.7 . When the orifice area ratio exceeds 0.7 , the flow rate increase is gently down. With the downstream orifice width at $86.6 \mu \mathrm{m}$, the flow rate is close to saturation. 


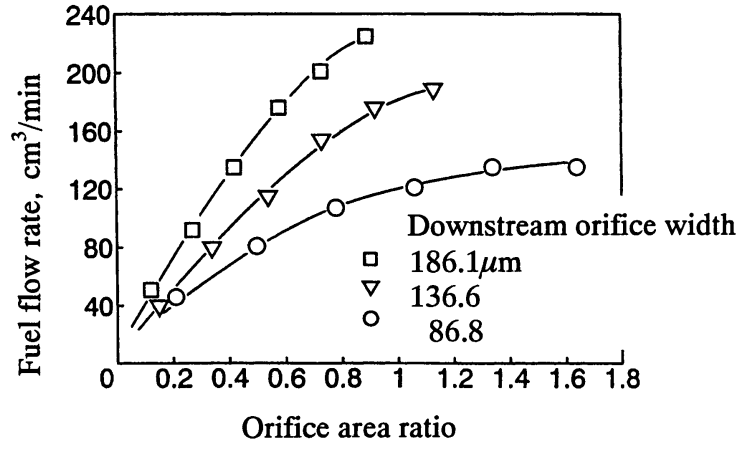

Fig. 8 Fuel flow rate characteriatics of combined SPN (injection pressure: $300 \mathrm{kPa}$ )

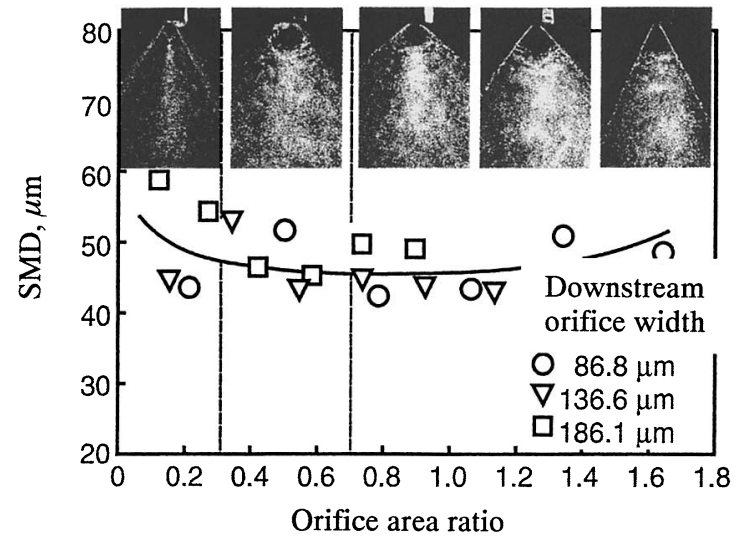

Fig. 9 SMD changes on orifice area ratio (injection pressure: $300 \mathrm{kPa}$ )

\subsection{Spray droplet size}

We measured the spray droplet size (Sauter Mean Diameter; SMD) produced by the combined SPN with various orifice area ratios. Figure 9 shows the result of the measured SMD, along with some typical spray patterns selected from those shown Fig. 7. As mentioned in section 4.1, the atomization performance decreases when the orifice area ratio is lower than 0.3 , and the fan spray angle decreases when the orifice area ratio exceeds 0.7 . The SMD measurement revealed that the spray droplet size increases when the orifice area ratio is lower than 0.3 or higher than 0.7. In other words, there exists an orifice area ratio range in which atomization becomes optimal. The pattern of SMD change with the orifice area ratio is almost the same for all downstream orifice widths.

As shown in Fig. 9, the SMD in the optimally atomized spray produced by the combined SPN is around $45 \mu \mathrm{m}$. An air-assist injector that adds air flow in the fuel has been put to practical use as an EFI injector with improved fuel atomization. Considering that the SMD of fuel spray by this injector is around $50 \mu \mathrm{m}$, it can be said that the combined SPN provides appreciably high atomization performance.

\section{Fan Spray Formation Mechanism}

We studied the mechanism of the fan spray formation

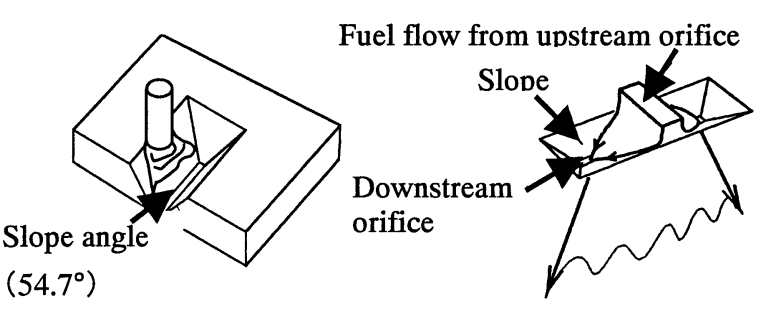

(a) Fuel impingement to nozzle wall (b) Fan spray formation

Fig. 10 Fan spray formation mechanism using combined SPN

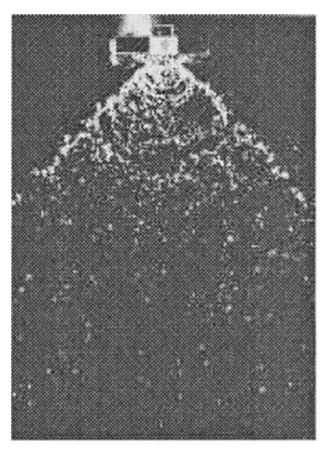

Slope angle: $40^{\circ}$

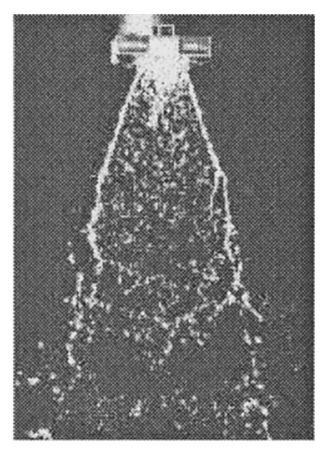

$70^{\circ}$
Fig. 11 Spray pattern change on slope angle

and characteristics described in section 4 .

As already described, the basic element of the combined SPN is a rectangular nozzle orifice surrounded by sloped walls on the four sides. Two such nozzle orifices are superposed one over the other in the combined SPN. The upstream orifice provides a passage that directs fuel flow toward the center of the downstream orifice. The fuel flowing through this passage collides against the two opposed longitudinal slopes of the downstream orifice, forming a film of liquid fuel that spreads laterally on each of these slopes. The liquid films then flow downward along the slopes toward the center and collide against each other. Due to this liquid-liquid collision, the liquid films further spread laterally along the longitudinal slopes, causing the fuel to exit the downstream orifice in the form of a thin, flat fan spray. This mechanism is schematically shown in Fig. 10 (a) and (b).

With the aim of verifying the abovementioned fan spray formation mechanism, we experimentally studied the relation between downstream-orifice slope angle and fan spray angle. Our expectation was that as the slope angle became smaller, the liquid fuel injected from the upstream orifice would spread more widely as it collided against the slope, and the liquid fuel flow would be diverted more largely, resulting in higher effect of the liquidliquid collision on increasing the fan spray angle. Since the orifice slope angle of SPN cannnot be changed, we fabricated the five times expanded metal models of the combined SPN. In the experiment, we used unleaded gasoline, which is the same fluid as is expected to be used in the combined SPN. Figure 11 shows the fan spray pat- 
terns obtained by the expanded metal models with different slope angles. As was expected, the fan spray angle increased with decrease in the downstream-orifice slope angle: it was $90^{\circ}$ when the slope angle was $40^{\circ}$ (left picture of Fig. 11), and $40^{\circ}$ when the slope angle was $70^{\circ}$ (right picture of Fig. 11).

Tanazawa et al. carried out a study regarding fan spray formation by liquid-liquid impingement ${ }^{(4)}$. To study the spray characteristics of an impingement nozzle, they experimentally examined the liquid film and spray pattern formed by impingement between liquid jets from two thin tubes arranged at an angle of $\alpha$ as shown in Fig. 12. Figure 13 shows some typical spray patterns formed by this method, which are cited from Ref. (4). The broken lines in the photographs were drawn by us, with the aim of making each spray pattern and spray angle more distinguishable.

Figure 14 compares the relation between impingement angle and spray angle obtained by Tanazawa et al., with those obtained by the expanded metal models and actual combined SPN.

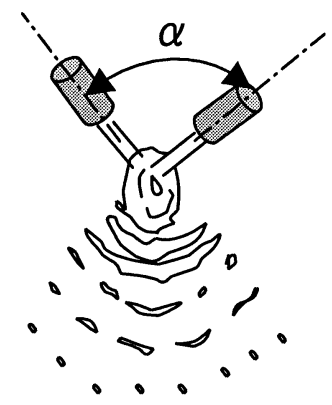

Fig. 12 Two liquid jets impingement with angle $\alpha$

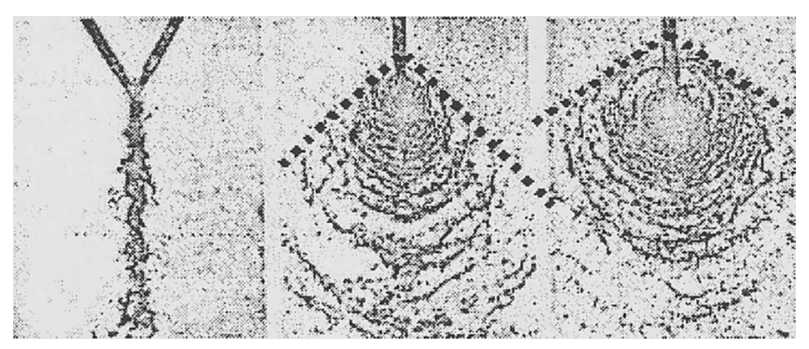

(a) $\alpha=60^{\circ}$

(b) $\alpha=90^{\circ}$

Fig. 13 Spray patterns formed by two liquid jets impingement $^{(4)}$

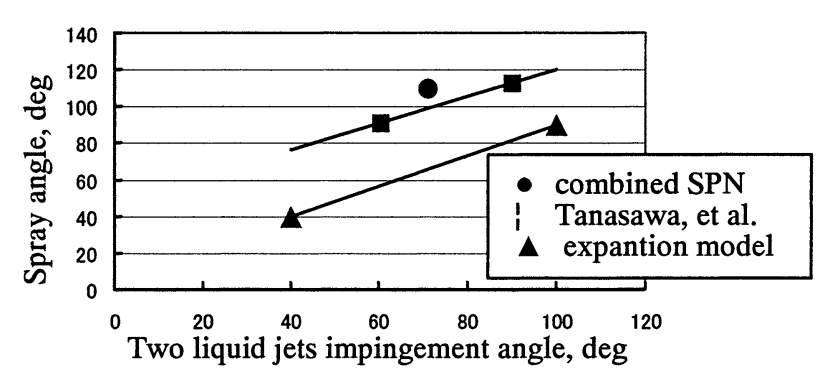

Fig. 14 Two liquid jets impigement angle, deg
The result by Tanazawa et al. is mostly consistent with our result with the combined SPN. With the expanded metal model, however, the spray angle is considerably smaller than the other two cases. The conditions for experiments with the expanded model were set so that the Reynolds number became equal to that for the combined SPN. Therefore, while the liquid flow rate in the expanded model was five times larger than that in the combined SPN, the liquid velocity at the nozzle orifice of the expanded model was $1 / 5$ of the combined SPN. In addition, since the orifice width of the expanded model was five times larger than that of the combined SPN, the thickness of liquid film sprayed was also five-fold larger. It is presumed that the low liquid velocity in the nozzle orifice, combined with greater gravitational influence on the liquid film, caused the small spray angle with the expanded model. However, the impingement and spray angles with the expanded model provide virtually the same proportionality relation as those by Tanazawa et al. This indicates that the expanded model is effective in confirming the fan spray formation mechanism in the orifice surrounded by sloped wall.

Closer observation of Fig. 14 reveals that the spray angle of the combined SPN is slightly larger than that described by Tanazawa et al. This is presumably due to the double-barreled effect of the liquid collision against the slope and the liquid-liquid impingement.

\section{Nozzle Flow Analysis for Combined SPN}

The preceding section clarified the fan spray formation mechanism of the combined SPN, but not the liquid flow status in the nozzle. The present section studies the liquid flow status in the combined SPN, by means of threedimensional numerical flow analysis.

Commercially available numerical analysis code STAR-CD was used. A 1/4 numerical model as shown in Fig. 15 was created as the target of this analysis.

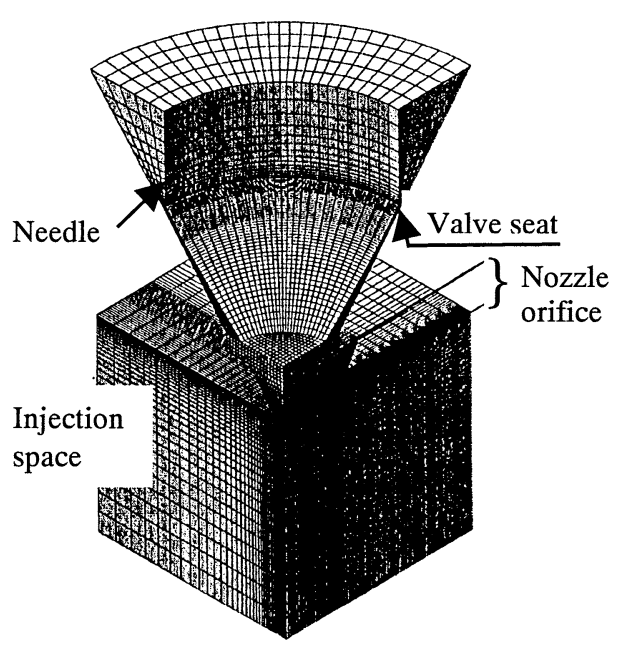

Fig. 15 Numerical model for nozzle flow analysis 


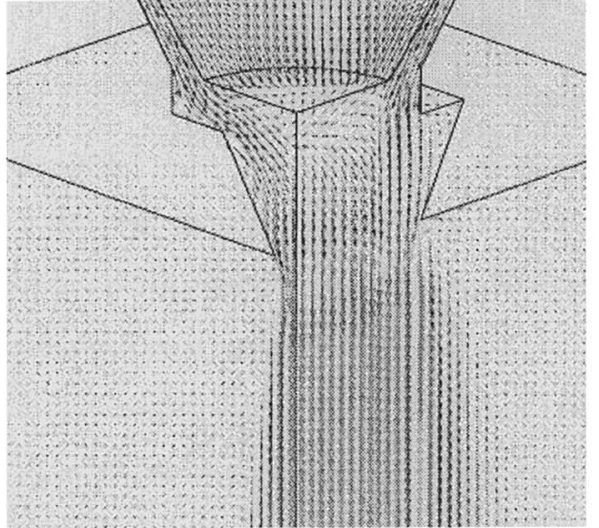

(a) Single SPN

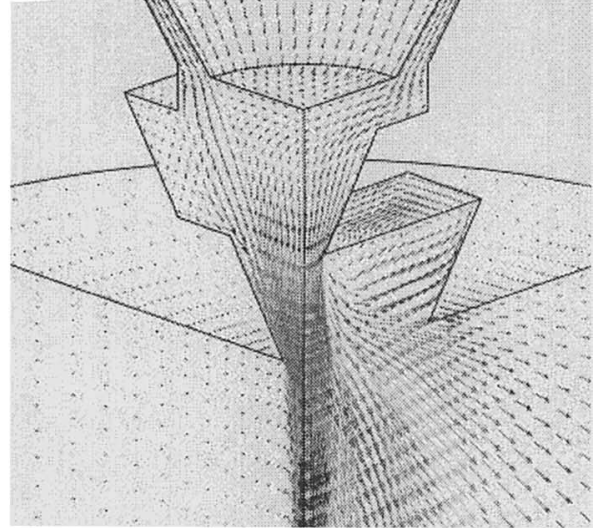

(b) Combined SPN

Fig. 16 Numerical flow analysis results for SPN

This $1 / 4$ model differs in configuration from the experimental assembly (shown in Fig. 4) in which the combined SPN is mounted on a nozzle holder for constantpressure injection; to enable design evaluation of the combined SPN for application to an actual injector, the $1 / 4$ model contained an injector needle arranged above the combined SPN. We decided that this needle would not have a significant influence on our numerical analysis, since the objective of the analysis was to observe the change in the fuel flow status, particularly in the downstream orifice of the combined SPN, and the change in spray pattern from the downstream orifice. To avoid any influence of the needle and to accurately grasp the flow change in the combined SPN, however, we studied the flow characteristic in detail by comparing the numerical analysis result for a single SPN (shown in Fig. 16 (a)) with that for the combined SPN (shown in Fig. 16(b)).

Unsteady-state single-phase flow calculation was carried out using two different conditions: one for a single SPN; the other for the combined SPN. Standard k- $\varepsilon$ model was used as the turbulence model. Figure 16 shows the results. To facilitate grasping the flow state, each vector in Fig. 16 mainly represents the direction of flow, and not the flow velocity; the velocity is represented by color. The flow velocity characteristic of the combined SPN is described in the following paragraph.

For a single SPN, the fluid flowing out of the needle is concentrated in the center of the SPN, and sprayed from the nozzle orifice almost in parallel with the central axis of the orifice, as shown in Fig. 16(a). The flow velocity near the needle and in the orifice is approximately $12 \mathrm{~m} / \mathrm{s}$. Although small vortexes are observed in the upper corner of the orifice, they do not have influence on the main flow. Most of the lines of the flow jetting out of the orifice are parallel with the central axis. The jet velocity is around $20 \mathrm{~m} / \mathrm{s}$. Only a few wake lines are observed around the main flow. The wake velocity is approximately $6 \mathrm{~m} / \mathrm{s}$.

For the combined SPN, the flow state in the upstream orifice is the same as in the orifice of the single SPN. The fluid flowing out of the upstream orifice enters the downstream orifice where it spreads laterally, and jets out in a fan shape. The flow velocity is about $18 \mathrm{~m} / \mathrm{s}$ at points around the central main flow, and is reduced at points farther from the center. At each edge region of the orifice, the flow velocity is $14 \mathrm{~m} / \mathrm{s}$.

The above analytical result confirms the following flow characteristics in the combined SPN. Liquid fuel flowing out of the upstream orifice collides against the downstream orifice's slopes where the flow is given lateral components, so that the liquid is sprayed obliquely in a fan-like form, out of the downstream orifice.

\section{Conclusions}

A new type of fuel injection nozzle has been developed by combining two silicon plate nozzles (SPNs) that are manufactured using micro-machining technology. The developed combined SPN produces a fan spray and provides superior atomization performance. The experimental studies for the fan spray formation mechanism and spray characteristics of the combined SPN have revealed the following.

( 1 ) The combined SPN forms a fan spray by the following mechanism. Liquid fuel flowing through the passage of the upstream orifice is directed toward the center of the downstream orifice and collides against the downstream orifice's opposed longitudinal slopes, spreading laterally and then downward along them. As the laterally spreading films of liquid flow downward on the slopes, they impinge against each other and further spread sideway. As a result, liquid fuel is sprayed in a flat fan shape from the downstream orifice.

(2) The spray pattern changes with the ratio of upstream orifice area to downstream orifice area. When the orifice area ratio is too small, the fuel flow rate from the upstream to downstream orifice will become too low, resulting in insufficient lateral spread of liquid film. When 
the orifice area ratio is too large, the liquid film will spread excessively in the lateral direction and sticks to the longitudinal end walls of the downstream orifice. The optimal orifice area ratio is between 0.3 and 0.7 .

(3) The fan spray angle of the combined SPN with an optimal orifice area ratio is slightly larger than that of the jet impingement nozzle already developed. Being capable of producing atomized spray with Sauter Mean Diameter (SMD) of approximately $45 \mu \mathrm{m}$, the combined SPN provides higher atomization performance than does the conventional air-assist injector.

\section{Acknowledgment}

We would like to express our gratitude to the following staff of the Silicon Device Research Section, Device Department, Toyota Central R\&D Lab., Inc. for their kind cooperation in our manufacturing of the silicon plate nozzles:

Mr. Susumu Sugiyama, the-then chief researcher (currently Professor at the College of Science and Engi- neering, Ritsumeikan University)

Mr. Osamu Tabata (currently Professor at the College of Science and Engineering, Ritsumeikan University)

Mr. Keiichi Shimaoka, assistant engineer

\section{References}

( 1 ) Sugimoto, T., Takeda, K. and Yoshizaki, H., Atomization of Air-Mix Type 2-Hole Injector for 4-Valve Engines, Proceedings of the 9th Symposium of Internal Combustion Engines, (in Japanese), (1991), pp.343348.

( 2 ) Tani, Y., Mori, Y., Mochizuki, K. and Suzuki, A., Fuel Atomization of a Multiple-Hole Nozzle Injector, SAE Paper No.2000-01-1428, (2000), pp.155-162.

( 3 ) Tani, Y., Inagaki, H., Saito, A. and Suzuki, T., Fuel Atomization Using Silicon Plate Nozzle, Trans. Jpn. Soc. Mech. Eng., (in Japanese), Vol.69, No.682, B (2003), pp.217-222.

(4) Tanasawa, Y., Sasaki, S. and Nagai, N., A Study on Impingement Nozzle for Diesel Engines, Tech. Report Tohoku Univ., Vol.22, No.2 (1958), pp.153-172. 